\title{
Analyzing the disease module associated with osteosarcoma via a network- and pathway-based approach
}

\author{
YI ZHANG $^{1}$ and FEI YANG ${ }^{2}$ \\ Departments of ${ }^{1}$ Orthopaedic Microsurgery and ${ }^{2}$ Orthopedic Joint Surgery, Central Hospital of Zibo, \\ Zibo, Shandong 255000, P.R. China
}

Received February 26, 2018; Accepted July 13, 2018

DOI: $10.3892 /$ etm.2018.6506

\begin{abstract}
Osteosarcoma is the most common type of primary malignant bone tumor observed in children and adolescents. The aim of the present study was to identify an osteosarcoma-related gene module (OSM) by looking for a dense module following the integration of signals from genome-wide association studies (GWAS) into the human protein-protein interaction (PPI) network. A dataset of somatic mutations in osteosarcoma was obtained from the $\mathrm{dbGaP}$ database and their testing P-values were incorporated into the PPI network from a recent study using the dmGWAS bioconductor package. An OSM containing 201 genes (OS genes) and 268 interactions, which were closely associated with immune response, intracellular signal transduction and cell activity was identified. Topological analysis of the OSM identified 11 genes, including APP, APPBP2, ATXN1, HSP90B1, IKZF1, KRTAP10-1, PAK1, PDPK1, SMAD4, SUZ12 and TP53 as potential diagnostic biomarkers for osteosarcoma. The overall survival analysis of osteosarcoma for those 11 genes based on a dataset from the Cancer Genome Atlas, identified APP, HSP90B1, SUZ12 and IKZF1 as osteosarcoma survival-related genes. The results of the present study should be helpful in understanding the diagnosis and treatment of osteosarcoma and its underlying mechanisms. In addition, the methodology used in the present study may be suitable for the analysis of other types of disease.
\end{abstract}

\section{Introduction}

Osteosarcoma is the most common histological form of primary bone cancer in children and adolescents (1). The incidence rates of osteosarcoma in Americans under the age of 20 are estimated to be about 5.0 per million per year for

Correspondence to: Dr Fei Yang, Department of Orthopedic Joint Surgery, Central Hospital of Zibo, 54 Gongqingtuanxi Road, Zhangdian, Zibo, Shandong 255000, P.R. China

E-mail: feiytj@outlook.com

Key words: osteosarcoma, dbGaP, network, disease module, protein-protein interaction the general population (1). It occurs more frequently in the metaphyseal region of tubular long bones, with $42 \%$ occurring in the femur, $19 \%$ in the tibia, and $10 \%$ in the humerus (1).

Due to complex nuclear and highly unstable genomes, osteosarcoma usually shows both numerical and structural chromosomal changes (2). Osteosarcoma often presents in Paget's disease of the bone, which may be caused by a mutation in the TNFRSF11A gene on chromosome 18q22 (3). Osteosarcoma is a characteristic of Li-Fraumeni syndrome-1 (LFS1), which is caused by a mutation of the TP53 gene, and of Li-Fraumeni syndrome-2 (LFS2), which is caused by a mutation of the CHEK2 gene. Sadikovic et al (4) used 10 pediatric osteosarcoma tissue samples for integrative whole-genome analysis of promoter methylation, gene expression, and DNA copy number. Consequently, hypomethylation, overexpression, and copy number gain were identified for the histone cluster 2 genes on chromosomes 1q21.1-q21.3. They also discovered i) the loss of chromosome 8p21.3-p21.2, ii) lower expression of TNFRSF10A, DOCK5, and TNFRSF10D genes, iii) copy number gain of chromosome 6p21.1-p12.3, and iv) amplification-related overexpression of RUNX2.

Currently, multidrug resistance (MDR) is an urgent problem to be solved in osteosarcoma treatment. The human MDR gene 1 (MDR1) reportedly plays an important role in the drug resistance process in osteosarcoma (5). A recent study shows that the expression of trichorhinophalangeal syndrome type 1 (Trps1) is directly related to MDR1/P-gp, and Trps1 can promote MDR1/P-gp gene expression in osteosarcoma cell lines. Therefore, Trps1 should be a promising molecular target for reversing drug resistance in osteosarcoma (6).

Although there has been some improvement in the pathogenesis, diagnosis, and treatment of osteosarcoma in recent years, the mechanism underlying the development of osteosarcoma remains obscure. Furthermore, efforts have rarely been intended at implementing system biology-based analyses to elaborate the underpinning pathological molecular mechanisms of osteosarcoma. Comprehensive analysis of latent causal genes within a pathway and/or a network framework could provide more holistic insights than classical single-gene analyses. In the present study, we first identified an osteosarcoma-related gene module (OSM) by combining the genome-wide association studies (GWAS) dataset of osteosarcoma with whole protein-protein interaction (PPI) network. Functions of genes contained in the OSM were also 
explored through WebGestalt and ToppGene online tools. Potential osteosarcoma diagnosis and treatment targets were further screened through topological and survival analyses of the OSM. This study should help understand the molecular mechanisms of osteosarcoma and provide a valuable pipeline for other types of disease.

\section{Materials and methods}

Data sources. Results from MutSigCV (broadinstitute. org/cancer/cga/mutsig) analysis on somatic mutations in osteosarcoma were obtained from dbGaP (ncbi.nlm.nih.gov/gap) (NCBI dbGaP study accession: phs000699.v1.p1 and dbGaP analysis accession: pha003862). The datasets consisted of 58 pairs of osteosarcoma and normal adjacent tissues. Forty-nine percent of the patients were male and $47 \%$ had metastases at diagnosis. Five-year overall survival was $49 \%$ with $33 \%$ for patients with metastatic disease and $64 \%$ for patients with localized disease. Illumina HiSeq 2000 was used for the whole genome sequencing of all samples. Genes with a P-value $<1$ were selected as seeds to identify the disease module underlying osteosarcoma.

The human PPI data containing 16,022 nodes and 228,122 edges were obtained from Hu's study (7), which collected and integrated two PPI datasets, i.e., Protein Interaction Network Analysis (PINA) platform (May 21, 2014) (8) and a human interactome compiled by a recent study (9).

Disease module identification. The OSM was identified using dmGWAS version 3.0 (10), an R package that implements heuristic local search algorithms to recognize candidate subnetworks or genes associated with complex diseases by incorporating the association signal from GWAS datasets into the PPI network, which has been efficiently applied in identifying disease modules. Osteosarcoma-related seed genes with their corresponding tested P-values and PPI networks were used as input, and other parameters were set as per the dmGWAS recommendations. Ultimately, the candidate disease modules were ranked in accordance with their normalized module scores and empirical P-values.

To test the non-randomness of the identified module, we first generated 1,000 random networks with the same number of nodes and edges as the identified OSM by utilizing the 'networkx' module in python (https://www.python.org/). Then, we computed the average values of clustering coefficients and the shortest-path distance for each random network. By counting the number of random networks with the average clustering coefficient $\left(\mathrm{N}_{\mathrm{CC}}\right)$ greater than that of the OSM as well as the number of random networks with the average shortest-path distance $\left(\mathrm{N}_{\mathrm{SD}}\right)$ less than that of the OSM, we could evaluate the significance level of non-randomness by calculating the empirical P-value $\left(\mathrm{N}_{\mathrm{CC}} / 1000\right.$ and $\left.\mathrm{N}_{\mathrm{SD}} / 1000\right)$.

Functional enrichment analysis of the OSM. The functional features of the OSM were examined through WebGestalt (11) and ToppGene (12). WebGestalt incorporates the information from multiple sources to detect the biological themes out of the given gene lists, including identifying the significantly enriched gene ontology (GO) terms. GO terms with a $\mathrm{P}<0.05$ were considered significantly enriched. ToppGene was chosen to analyze the Kyoto Encyclopedia of Genes and Genomes (KEGG) pathways enriched in the OSM. The pathways with false discovery rate (FDR) $<0.05$ were considered significantly enriched.

Pathway crosstalk analysis of the significantly enriched pathways. Further, we carried out pathway crosstalk analysis to investigate the interactions among significantly enriched pathways (13). To demonstrate the overlap between any pair of pathways, we adopted two measurements, i.e., the Overlap Coefficient

$$
(\mathrm{OC})=\frac{|A \cap B|}{\min (|A|,|B|)}
$$

and the Jaccard Coefficient

$$
(\mathrm{JC})=\mid \frac{A \cap B}{A \cup B}
$$

where $A$ and $B$ denote the lists of genes contained in the two tested pathways. We carried out the following procedure to establish the pathway crosstalk: i) We chose a set of pathways with FDR $<0.05$ for crosstalk analysis; ii) we calculated the number of shared OS genes between any pair of pathways. Pathway pairs with $<2$ overlapped OS genes were discarded; iii) we computed the overlap of all pairs of pathways and ranked them in accordance with their OC and JC values; and iv) we used the Cytoscape (14) software to visualize the chosen pathway crosstalk.

Inferring essential genes in the OSM. Previous studies reported that hub genes (high degree) and bottleneck genes (high betweenness) in gene networks are more important for cell survival (15-17). In addition, another study reported that minimum dominating sets of proteins (MDSets) play a topologically and biologically important role in controllability in protein interaction networks (18). Briefly, MDSet is an optimized subset of nodes from where each remaining node can be immediately reached by a single interaction. To identify the essential genes in the OSM, we ranked all module genes on the basis of their degree and betweenness centrality and obtained the MDSets within the OSM. Overlapping genes among hub genes, bottleneck genes, and genes contained in the MDSets were considered valuable for osteosarcoma progression.

Statistical analysis. All of the statistical analyses were conducted in R 3.4.1 (r-project.org/) and Python 2.7.11 (python. org/downloads/release/python-2711/). Networkx module of Python was used for the non-randomness test of identified gene module. $\mathrm{P}<0.05$ was used as the criteria for the identification of significantly enriched functions.

\section{Results}

Identification of disease module associated withosteosarcoma. Fig. 1 illustrates the flow chart of this study. By taking advantage of dmGWAS (10), candidate disease modules related to 


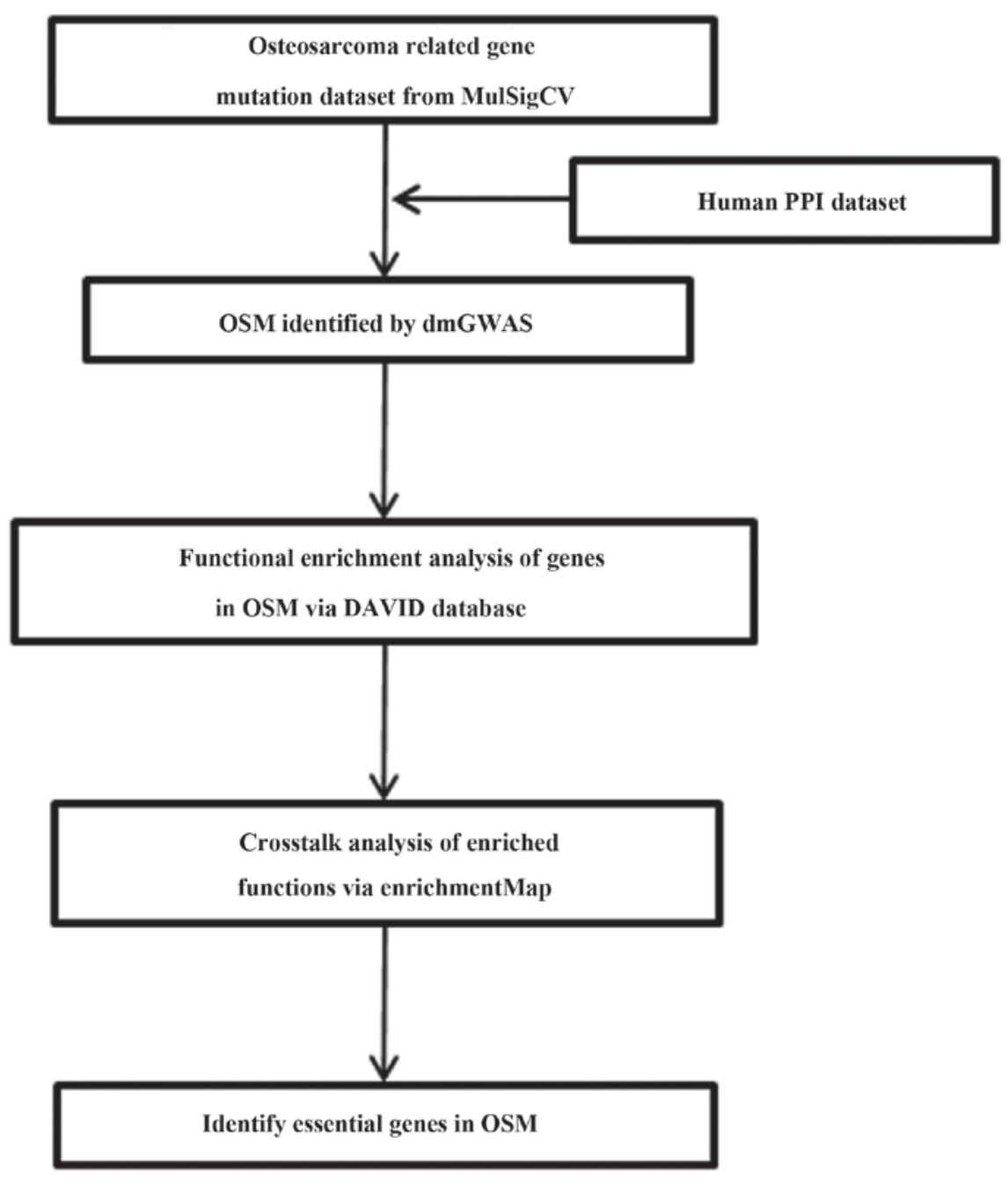

Figure 1. Flow chart of the study. OSM, osteosarcoma-related gene module; PPI, protein-protein interaction; GWAS, genome-wide association studies.

osteosarcoma were deduced by incorporating the association signal from GWAS into the PPI network. The top 150 candidate modules with the highest module scores and empirical $\mathrm{P}<0.001$ were selected. The OSM was obtained by merging these modules and excluding the redundant entries, which resulted in a subnetwork containing 201 nodes and 268 edges (Fig. 2). To test for the non-randomness of the identified disease module, 1,000 random subnetworks were generated, and their corresponding average clustering coefficient and the mean shortest-path distance were compared with the corresponding values of the OSM. For these random subnetworks, the average clustering coefficient was 0.01 , which is statistically significantly smaller than that of the OSM (clustering coefficient, 0.07; empirical $\mathrm{P}<0.001$ ). The average shortest-path distance for the 1000 random subnetwork was 4.75, which was statistically significantly higher than that of the OSM (shortest-path distance, 3.37; empirical $\mathrm{P}<0.001$ ). This indicated that our identified OSM was a non-random network. Notably, some of the genes within the OSM, such as small nuclear ribonucleoprotein U11/U12 subunit 25 (SNRNP25) $(19,20)$, cyclin B3 (CCNB3) (21), tumor protein p53 (TP53) $(22,23)$, OS9, endoplasmic reticulum lectin (OS9) (24), and phosphatase and tensin homolog (PTEN) (25), have been reportedly associated with osteosarcoma in previous studies.
Significantly enriched functions of the OSM. In this study, significantly enriched GO terms and KEGG pathways of the OSM were identified through WebGestalt and ToppGene online tools, respectively. Consequently, a total of $30 \mathrm{GO}$ terms (Table I) and 32 KEGG pathways (Table II) were significantly associated with the OSM. GO terms related to response to stimulus (e.g., response to estradiol and to epidermal growth factor) $(26,27)$ and intracellular signal transduction (e.g., hippo signaling) (28) were obtained in this study; these were consistent with the previous findings about osteosarcoma. Terms directly related to protein or nucleic acid binding (e.g., RNA polymerase II transcription factor binding, protein N-terminus binding, lipoprotein particle receptor binding, single-stranded RNA binding, and damaged DNA binding) and cataly tic activity (e.g., deacetylase activity, phosphotransferase activity, phosphate group as a acceptor, and kinase activity of nucleobase-containing compounds) were also included. GO terms related to translation (e.g., cytoplasmic translation) were also enriched in OS genes. In line with previous studies, several pathways, such as mTOR signaling pathway (ranked 5th) $(29,30)$, Hippo signaling pathway (ranked 9th) (31), PI3K-Akt signaling pathway (ranked 10th) $(30,32)$, MAPK signaling pathway (ranked 11th) (33), Wnt signaling pathway (ranked 22nd) (34,35), p53 


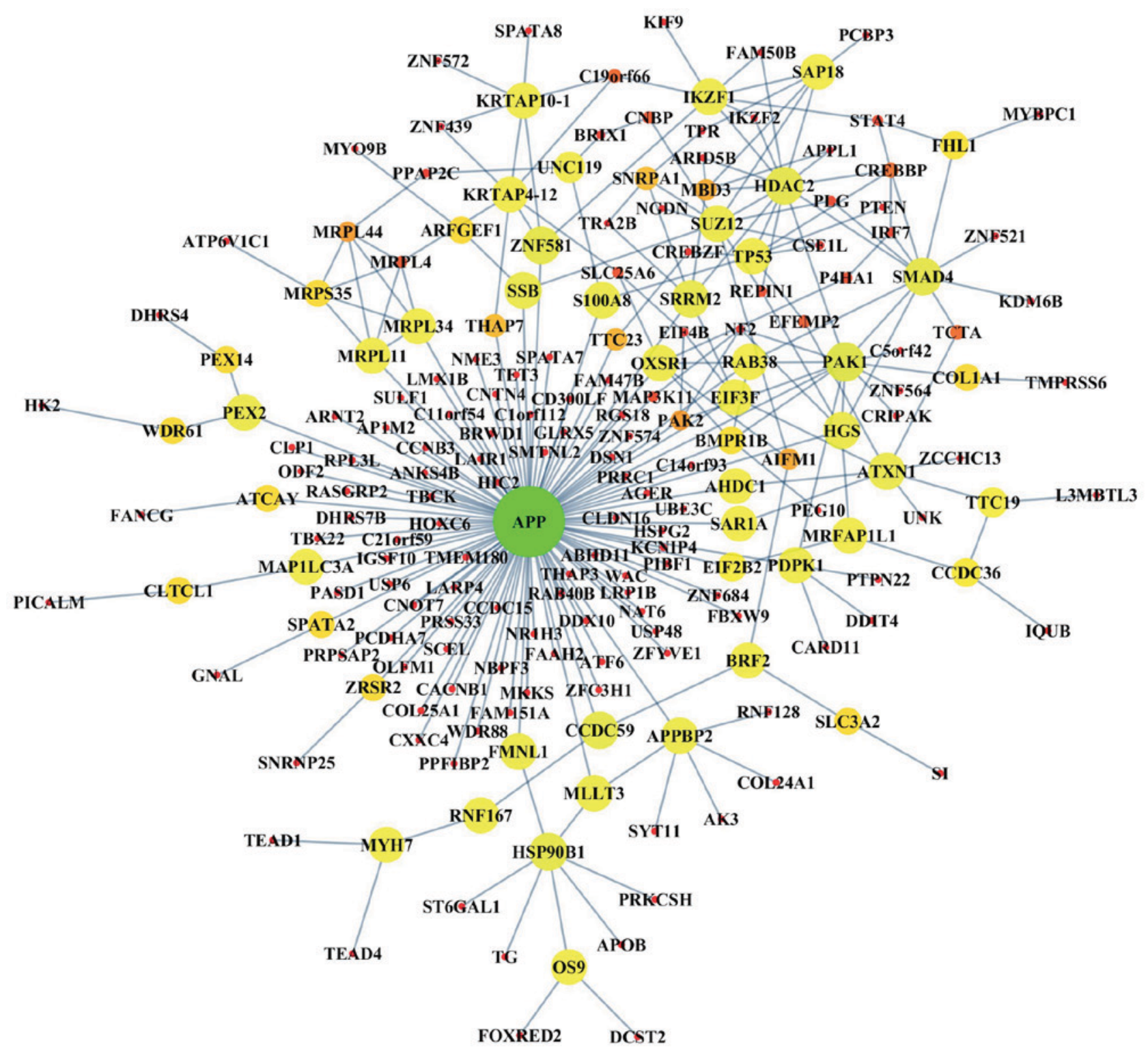

Figure 2. Osteosarcoma-specific network. An osteosarcoma-specific network was constructed by integrating the association signal from GWAS into the protein-protein interaction network with 201 nodes and 268 edges. Node size corresponds to its degree in the OSM. A larger size indicates a higher degree, and the node color corresponds to its betweenness centrality in the OSM. Brighter colors indicate higher betweenness centrality. OSM, osteosarcoma-related gene module; GWAS, genome-wide association studies.

signaling pathway (ranked 24th) (36-38), and TGF- $\beta$ signaling pathway (ranked 27th) $(38,39)$, were enriched in OS genes. In addition, several cancer-related pathways were identified, such as Pathways in cancer, Proteoglycans in cancer, central carbon metabolism in cancer, and transcriptional misregulation in cancer. Moreover, cell proliferation, survival, and apoptosis-associated biological processes comprising cell cycle, FoxO signaling pathway, protein processing in the endoplasmic reticulum, RNA transport, and focal adhesion were also obtained.

Crosstalk among significantly enriched pathway. To understand how significantly enriched pathways interact with each other, we carried out a pathway crosstalk analysis among the 32 significantly enriched pathways. The approach was based on the assumption that two pathways were considered to interact with each other if they shared a proportion of the OS genes. With this criterion for pathway crosstalk analysis, 22 pathways were selected. All the pathway pairs were used to build the pathway crosstalk, and the overlapping level between two pathways was measured on the basis of the average scores of the coefficients OC and JC. Based on their crosstalk, these pathways could be roughly divided into two main modules, each of which included pathways that shared more interactions compared with other pathways and might involve the same or similar biological processes (Fig. 3). One module primarily comprised cell growth, death, and cancer-related pathways, such as those associated with the cell cycle, p53 signaling pathway, central carbon metabolism in cancer, transcriptional misregulation in cancer, and chronic myeloid leukemia. Conversely, the second module was mainly dominated by signal transduction-related pathways, including mTOR signaling pathway, FoxO signaling pathway, Hippo signaling pathway, PI3K-Akt signaling 
Table I. Gene ontology terms enriched in the osteosarcoma-related gene module.

\begin{tabular}{|c|c|c|c|c|}
\hline Category & GO terms & Cell process & P-value & Genes included in the GO term \\
\hline Biological process & GO:0070482 & Response to oxygen levels & $1.43 \times 10^{-4}$ & $\begin{array}{l}\text { COL1A1; CREBBP; AGER; HDAC2; SMAD4; } \\
\text { PAK1; DDIT4; PTEN; TP53; HSP90B1; AIFM1; } \\
\text { ARNT2 }\end{array}$ \\
\hline Biological process & GO:0048511 & Rhythmic process & $2.15 \times 10^{-3}$ & $\begin{array}{l}\text { NR1H3; CREBBP; PASD1; FANCG; HDAC2; } \\
\text { SMAD4; PTEN; BMPR1B; TP53; EIF2B2 }\end{array}$ \\
\hline Biological process & GO:0032355 & Response to estradiol & $2.19 \times 10^{-3}$ & COL1A1; APOB; MBD3; PTEN; AIFM1; ARNT2 \\
\hline Biological process & GO:0002181 & Cytoplasmic translation & $3.02 \times 10^{-3}$ & EIF4B; SSB; UNK; EIF3F \\
\hline Biological process & GO:0031349 & $\begin{array}{l}\text { Positive regulation of } \\
\text { defense response }\end{array}$ & $4.05 \times 10^{-3}$ & $\begin{array}{l}\text { NR1H3; CREBBP; PTPN22; SLC25A6; IRF7; } \\
\text { PAK1;PAK2; PDPK1; S100A8; HSP90B1; CARD11 }\end{array}$ \\
\hline Biological process & GO:0071826 & $\begin{array}{l}\text { Ribonucleoprotein complex } \\
\text { subunit organization }\end{array}$ & $6.05 \times 10^{-3}$ & $\begin{array}{l}\text { CLP1; EIF4B; CNOT7; BRIX1; RPL3L; } \\
\text { MRPL11; ZRSR2 }\end{array}$ \\
\hline Biological process & GO:0035329 & Hippo signaling & $6.46 \times 10^{-3}$ & NF2; TEAD1; TEAD4 \\
\hline Biological process & GO:0055076 & $\begin{array}{l}\text { Transition metal ion } \\
\text { homeostasis }\end{array}$ & $7.64 \times 10^{-3}$ & TMPRSS6; APP; SMAD4; S100A8; PICALM \\
\hline Biological process & GO:0016570 & Histone modification & $7.68 \times 10^{-3}$ & $\begin{array}{l}\text { SAP18; CREBBP; KDM6B; SUZ12; HDAC2; } \\
\text { SMAD4; WAC; MBD3; TP53; WDR61; ARID5B }\end{array}$ \\
\hline Biological process & GO:0070849 & $\begin{array}{l}\text { Response to epidermal } \\
\text { growth factor }\end{array}$ & $8.13 \times 10^{-3}$ & COL1A1; PDPK1; TPR \\
\hline Molecular function & GO:0001085 & $\begin{array}{l}\text { RNA polymerase II } \\
\text { transcription factor binding }\end{array}$ & $1.13 \times 10^{-4}$ & $\begin{array}{l}\text { CREBBP; HDAC2; SMAD4; TEAD1; TEAD4; } \\
\text { TP53; MKKS }\end{array}$ \\
\hline Molecular function & GO:0019205 & $\begin{array}{l}\text { Nucleobase-containing } \\
\text { compound kinase activity }\end{array}$ & $1.31 \times 10^{-3}$ & CLP1; NME3; AK3; CARD11 \\
\hline Molecular function & GO:0005057 & $\begin{array}{l}\text { Signal transducer activity, } \\
\text { downstream of receptor }\end{array}$ & $3.19 \times 10^{-3}$ & $\begin{array}{l}\text { SMAD4; MAP3K11; PAK1; PAK2; BMPR1B; } \\
\text { OXSR1 }\end{array}$ \\
\hline Molecular function & GO:0003727 & Single-stranded RNA binding & $5.23 \times 10^{-3}$ & EIF4B; ATXN1; TRA2B; CNBP \\
\hline Molecular function & GO:0016776 & $\begin{array}{l}\text { Phosphotransferase activity, } \\
\text { phosphate group as acceptor }\end{array}$ & $7.33 \times 10^{-3}$ & NME3; AK3; CARD11 \\
\hline Molecular function & GO:0047485 & Protein N-terminus binding & $1.61 \times 10^{-2}$ & SRRM2; PEX14; TP53; THAP7 \\
\hline Molecular function & GO:0019213 & Deacetylase activity & $1.67 \times 10^{-2}$ & SAP18; HDAC2; MBD3 \\
\hline Molecular function & GO:0070325 & $\begin{array}{l}\text { Lipoprotein particle } \\
\text { receptor binding }\end{array}$ & $1.84 \times 10^{-2}$ & APOB; HSP90B1 \\
\hline Molecular function & GO:0003735 & $\begin{array}{l}\text { Structural constituent of } \\
\text { ribosome }\end{array}$ & $2.10 \times 10^{-2}$ & MRPL4; MRPS35; RPL3L; MRPL34; MRPL11 \\
\hline Molecular function & GO:0003684 & Damaged DNA binding & $2.30 \times 10^{-2}$ & CREBBP; FANCG; TP53 \\
\hline Cellular component & GO:0005788 & $\begin{array}{l}\text { Endoplasmic reticulum } \\
\text { lumen }\end{array}$ & $1.07 \times 10^{-3}$ & $\begin{array}{l}\text { OS9; COL1A1; COL24A1; APOB; P4HA1; } \\
\text { PRKCSH; HSP90B1; FOXRED2; COL25A1 }\end{array}$ \\
\hline Cellular component & GO:0000118 & Histone deacetylase complex & $7.72 \times 10^{-3}$ & SAP18; APPL1; HDAC2; MBD3 \\
\hline Cellular component & GO:0070603 & $\begin{array}{l}\text { SWI/SNF superfamily-type } \\
\text { complex }\end{array}$ & $1.43 \times 10^{-2}$ & SUZ12; APPL1; HDAC2; MBD3 \\
\hline Cellular component & GO:0017053 & $\begin{array}{l}\text { Transcriptional repressor } \\
\text { complex }\end{array}$ & $2.46 \times 10^{-2}$ & PASD1; APPL1; HDAC2; MBD3 \\
\hline Cellular component & GO:0005840 & Ribosome & $2.86 \times 10^{-2}$ & $\begin{array}{l}\text { REPIN1; MRPL4; MRPS35; RPL3L; MRPL34; } \\
\text { MRPL11; MRPL44 }\end{array}$ \\
\hline Cellular component & GO:0008023 & $\begin{array}{l}\text { Transcription elongation } \\
\text { factor complex }\end{array}$ & $2.90 \times 10^{-2}$ & MLLT3; PEX2; WDR61 \\
\hline Cellular component & GO:0044454 & Nuclear chromosome part & $4.17 \times 10^{-2}$ & $\begin{array}{l}\text { NR1H3; IKZF1; CREBBP; SUZ12; REPIN1; } \\
\text { HDAC2; SMAD4; MBD3; SSB; TP53; DSN1 }\end{array}$ \\
\hline Cellular component & GO:0031983 & Vesicle lumen & $4.48 \times 10^{-2}$ & APOB; APP; PLG; HSP90B1 \\
\hline Cellular component & GO:0030139 & Endocytic vesicle & $4.52 \times 10^{-2}$ & $\begin{array}{l}\text { AP1M2; SYT11; RAB38; APOB; HSP90B1; } \\
\text { FMNL1; PICALM }\end{array}$ \\
\hline Cellular component & GO:0031984 & Organelle subcompartment & $4.77 \times 10^{-2}$ & $\begin{array}{l}\text { AP1M2; ARFGEF1; SULF1; RAB38; APP; } \\
\text { ZFYVE1; ST6GAL1; CLTCL1 }\end{array}$ \\
\hline
\end{tabular}


Table II. Kyoto Encyclopedia of Genes and Genomes pathways enriched in the osteosarcoma-related gene module.

False

Pathways

P-value discovery rate

Genes included in the pathway

\begin{tabular}{|c|c|c|c|}
\hline Hepatitis B & $1.01 \times 10^{-6}$ & $2.47 \times 10^{-4}$ & STAT4, TP53, SMAD4, HSPG2, PTEN, IRF7, CREBBP \\
\hline Pathways in cancer & $1.67 \times 10^{-6}$ & $2.15 \times 10^{-3}$ & $\begin{array}{l}\text { RASGRP2, CREBBP, HDAC2, TP53, APPL1, SMAD4, } \\
\text { HSP90B1, PTEN, TPR, ARNT2 }\end{array}$ \\
\hline Prostate cancer & $1.80 \times 10^{-5}$ & $1.78 \times 10^{-3}$ & PTEN, TP53, CREBBP, HSP90B 1, PDPK1 \\
\hline Hepatitis C & $1.23 \times 10^{-4}$ & $4.72 \times 10^{-3}$ & CLDN16, PDPK1, IRF7, TP53, NR1H3 \\
\hline mTOR signaling pathway & $6.68 \times 10^{-5}$ & $4.72 \times 10^{-3}$ & PTEN, PDPK1, DDIT4, EIF4B \\
\hline Cell cycle & $8.84 \times 10^{-5}$ & $4.72 \times 10^{-3}$ & SMAD4, CCNB3, HDAC2, TP53, CREBBP \\
\hline Renal cell carcinoma & $9.72 \times 10^{-5}$ & $4.72 \times 10^{-3}$ & CREBBP, ARNT2, PAK2, PAK1 \\
\hline FoxO signaling pathway & $1.28 \times 10^{-4}$ & $4.72 \times 10^{-3}$ & SMAD4, PTEN, CCNB3, CREBBP, PDPK1 \\
\hline Hippo signaling pathway & $2.44 \times 10^{-4}$ & $7.23 \times 10^{-3}$ & SMAD4, BMPR1B, NF2, TEAD1, TEAD4 \\
\hline PI3K-Akt signaling pathway & $2.38 \times 10^{-4}$ & $7.23 \times 10^{-3}$ & $\begin{array}{l}\text { DDIT4, HSP90B1, PTEN, PDPK1, COL1A1, TP53, } \\
\text { EIF4B }\end{array}$ \\
\hline MAPK signaling pathway & $3.29 \times 10^{-4}$ & $8.85 \times 10^{-3}$ & RASGRP2, TP53, CACNB1, MAP3K11, PAK1, PAK2 \\
\hline Protein processing in endoplasmic reticulum & $3.75 \times 10^{-4}$ & $9.24 \times 10^{-3}$ & OS9, SAR1A, PRKCSH, HSP90B1, ATF6 \\
\hline RNA transport & $4.06 \times 10^{-4}$ & $9.25 \times 10^{-3}$ & EIF3F, EIF2B2, TPR, SAP18, EIF4B \\
\hline $\mathrm{T}$ cell receptor signaling pathway & $5.59 \times 10^{-4}$ & $1.18 \times 10^{-2}$ & PDPK1, CARD11, PAK1, PAK2 \\
\hline Huntington's disease & $6.85 \times 10^{-4}$ & $1.35 \times 10^{-2}$ & CLTCL1, HDAC2, TP53, SLC25A6, CREBBP \\
\hline Thyroid hormone signaling pathway & $8.98 \times 10^{-4}$ & $1.40 \times 10^{-2}$ & PDPK1, HDAC2, TP53, CREBBP \\
\hline Endometrial cancer & $8.92 \times 10^{-4}$ & $1.40 \times 10^{-2}$ & PTEN, TP53, PDPK1 \\
\hline Focal adhesion & $8.41 \times 10^{-4}$ & $1.40 \times 10^{-2}$ & PTEN, PDPK1, PAK1, PAK2, COL1A1 \\
\hline Proteoglycans in cancer & $8.60 \times 10^{-4}$ & $1.40 \times 10^{-2}$ & PDPK1, HSPG2, TP53, PAK1, EIF4B \\
\hline Colorectal cancer & $1.49 \times 10^{-3}$ & $2.20 \times 10^{-2}$ & SMAD4, APPL1, TP53 \\
\hline Ribosome & $1.56 \times 10^{-3}$ & $2.20 \times 10^{-2}$ & MRPL11, MRPL34, MRPL4, RPL3L \\
\hline Wnt signaling pathway & $1.83 \times 10^{-3}$ & $2.39 \times 10^{-2}$ & SMAD4, TP53, CREBBP, CXXC4 \\
\hline Central carbon metabolism in cancer & $1.86 \times 10^{-3}$ & $2.39 \times 10^{-2}$ & HK2, PTEN, TP53 \\
\hline p53 signaling pathway & $2.02 \times 10^{-3}$ & $2.50 \times 10^{-2}$ & CCNB3, PTEN, TP53 \\
\hline Chronic myeloid leukemia & $2.38 \times 10^{-3}$ & $2.82 \times 10^{-2}$ & SMAD4, HDAC2, TP53 \\
\hline Peroxisome & $3.42 \times 10^{-3}$ & $3.89 \times 10^{-2}$ & DHRS4, PEX2, PEX14 \\
\hline TGF- $\beta$ signaling pathway & $3.54 \times 10^{-3}$ & $3.89 \times 10^{-2}$ & SMAD4, BMPR1B, CREBBP \\
\hline Influenza A & $3.78 \times 10^{-3}$ & $3.99 \times 10^{-2}$ & PLG, IRF7, SLC25A6, CREBBP \\
\hline Protein digestion and absorption & $4.30 \times 10^{-3}$ & $4.24 \times 10^{-2}$ & SLC3A2, COL24A1, COL1A1 \\
\hline Transcriptional misregulation in cancer & $4.18 \times 10^{-3}$ & $4.24 \times 10^{-2}$ & HDAC2, TP53, ARNT2, MLLT3 \\
\hline Thyroid cancer & $4.95 \times 10^{-3}$ & $4.73 \times 10^{-2}$ & TP53, TPR \\
\hline Galactose metabolism & $5.29 \times 10^{-3}$ & $4.89 \times 10^{-2}$ & $\mathrm{HK} 2, \mathrm{SI}$ \\
\hline
\end{tabular}

pathway, Wnt signaling pathway, and MAPK signaling pathway. Furthermore, these two modules were linked by a couple of pathway interactions.

Essential genes in the OSM. To identify the essential genes within the OSM, we first ranked the OS genes based on their degree as well as betweenness centrality and obtained the MDSets simultaneously. The genes intersecting among these three gene lists were considered as essential OS genes, which included APP, APPBP2, ATXN1, HSP90B1, IKZF1, KRTAP10-1, PAK1, PDPK1, SMAD4, SUZ12, and TP53. Some of these genes, such as HSP90B1 (30,39), PAK1 (40), SMAD4 (31,41), and TP53 $(22,23)$, have already been reported to be associated with osteosarcoma. Besides, PDPK1 plays an important role in the mTOR signaling pathway and PI3K-Akt signaling pathway, which are reportedly involved in the pathogenesis of osteosarcoma. Kaplan-Meier analysis for overall survival with osteosarcoma and the expression values of essential genes from the Cancer Genome Atlas (TCGA) identified APP, HSP90B1, SUZ12, and IKZF1 as prognosis-related genes (Fig. 4). High expressions of APP (median survival for low and high APP expression patients is 95 and 64 months), HSP90B1 (median survival for low and high HSP90B1 expression patients is 90 and 60 months), and SUZ12 (median survival for low and high SUZ12 expression patients is 97 and 64 months) were significantly associated with shorter overall survival with osteosarcoma, whereas high expression of IKZF1 (median survival for low and high APP expression patients is 57 and 81 months) was significantly associated with longer overall survival with osteosarcoma. 


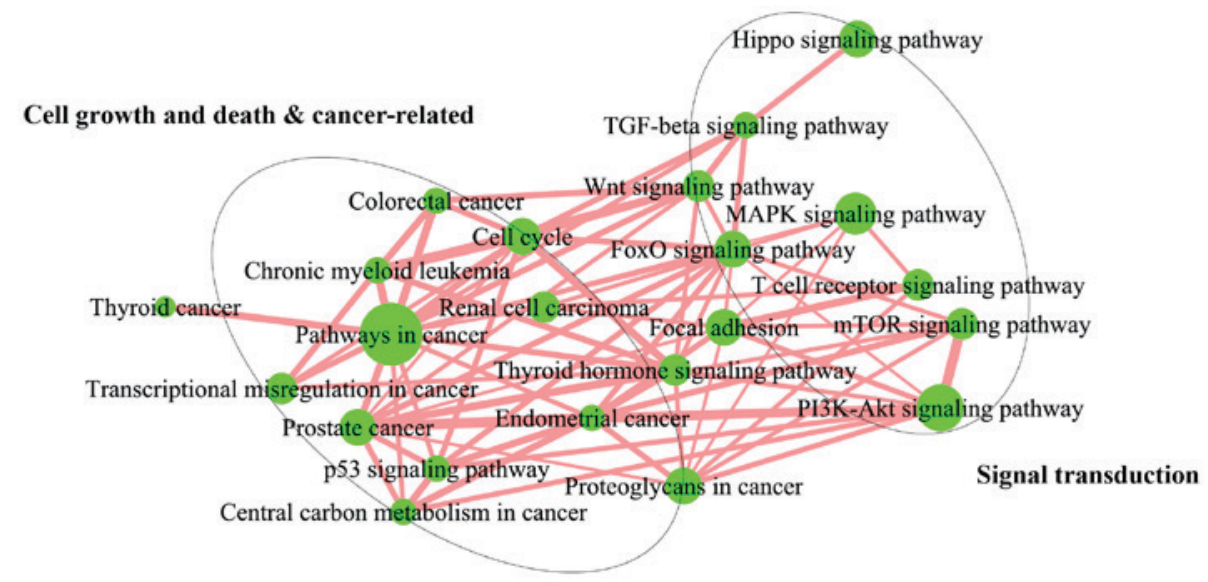

Figure 3. Pathway crosstalk among OS gene-enriched pathways. Nodes represent pathways, and edges represent crosstalk between pathways. Edge-width corresponds to the score of a specific pathway pair. A larger edge-width indicates a higher score, and the node size corresponds to the number of OS genes contained in the corresponding pathway.
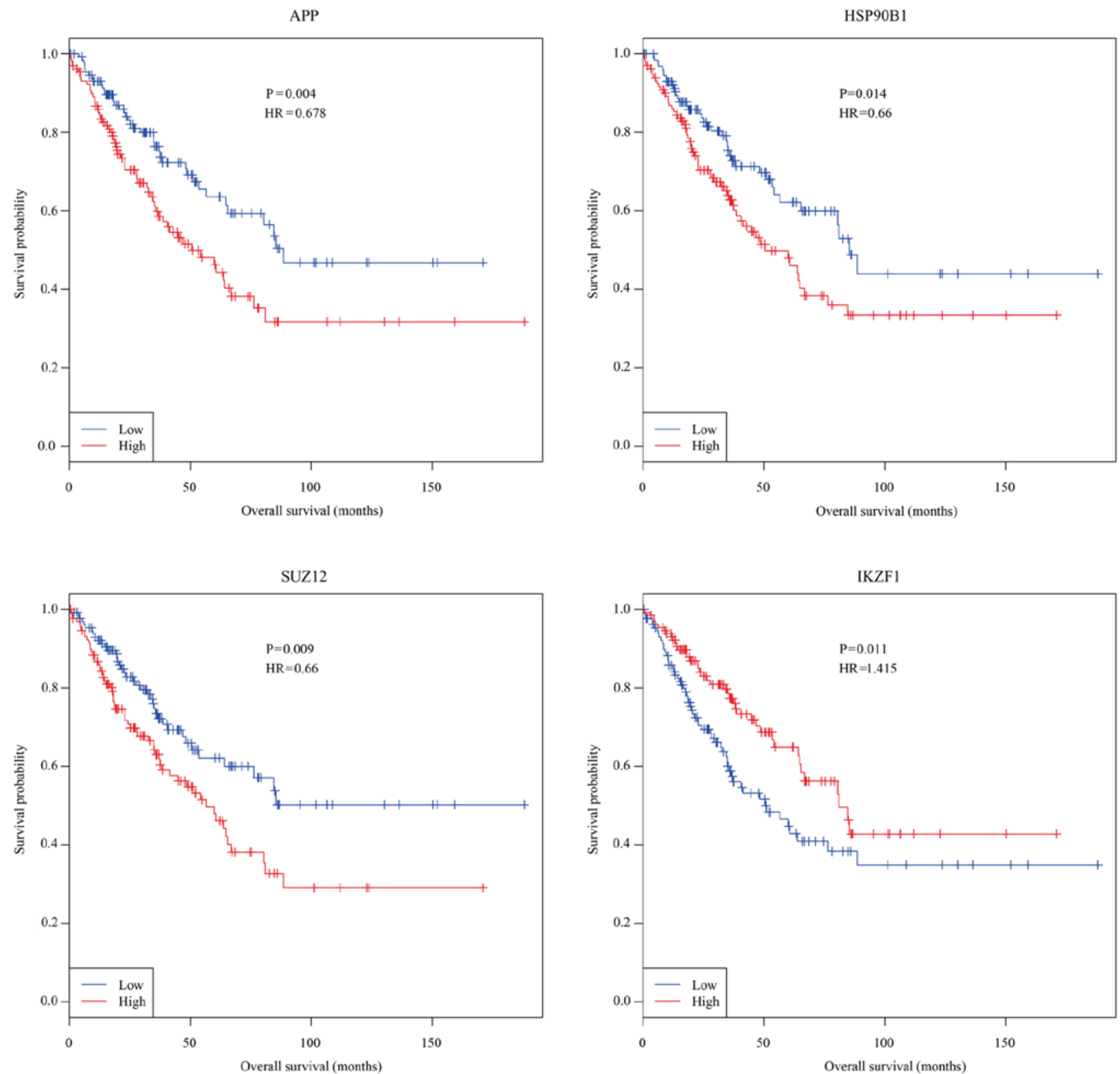

Figure 4. Kaplan-Meier curve analysis of the essential OS genes for overall survival of patients with osteosarcoma. Four essential OS genes were presented, including APP, HSP90B1, SUZ12 and IKZF1. HR, hazard ratio (low vs. high). 


\section{Discussion}

With the development of high-throughput technology, more and more genes/proteins have been identified as associated with osteosarcoma. Over the past ten years, much has been learnt from studies on animals, human subjects, or cell models about the molecular mechanisms underlying osteosarcoma. However, a comprehensive understanding of the biological process associated with osteosarcoma pathogenesis at the molecular level remains largely unclear. It is imperative to decipher the latent pathogenesis of osteosarcoma at the systems biology level. In this work, we first identified an OSM by combining the GWAS dataset of osteosarcoma with whole PPI network. A relatively comprehensive human physical interactome that integrated PPI data from various sources has been used in this study to reduce the limitations due to incomplete and noisy human interactome. Functions of genes contained in the OSM and potential osteosarcoma diagnosis and treatment targets were further explored.

The OSM we identified was a non-random network and 11 essential OS genes have been identified as essential in the module. Some of the essential in the module, such as HSP90B1 $(29,38)$, PAK1 (39), SMAD4 $(30,40)$, and TP53 $(21,22)$, have already been reported to be related to osteosarcoma, suggesting the reliability of the methods we used in this study. In addition, although several OS genes have been found as not directly involved in the pathogenesis of osteosarcoma, they are a part of biological pathways that play important roles in osteosarcoma development. PDPK1 plays an important role in the mTOR signaling pathway and PI3K-Akt signaling pathway, which are reportedly involved in the pathogenesis of osteosarcoma. DDIT4 and EIF4B were included in the OSM; both of them are involved in mTOR signaling pathway and PI3K-Akt signaling pathway, which are both closely associated with osteosarcoma. Some of the genes, such as STAT4 and LRP1B, have not been demonstrated to be related to osteosarcoma. Some of the members of the same family with those unproved genes, such as STAT3 $(42,43)$ and LRP1 (19), have shown their relevance in osteosarcoma in recent studies.

Kaplan-Meier curve analysis was performed to explore the associations between overall survival and these 11 essential OS genes in patients with osteosarcoma. Consequently, four essential OS genes, namely APP, HSP90B1, SUZ12, and IKZF1, were found to be significantly associated with overall survival with osteosarcoma $(\mathrm{P}<0.05)$. Importantly, SUZ12 polycomb repressive complex 2 subunit (SUZ12) has been identified at the breakpoints of a recurrent chromosomal translocation reported in endometrial stromal sarcoma. Thus, the OS genes identified as essential are a list of potential candidates and are reliable for researchers for further exploration.

In conclusion, our study provides a pipeline for systematically analyzing genome-wide datasets of osteosarcoma. Several valuable biomarkers which should be helpful for understanding the underlying mechanisms of osteosarcoma were identified.

\section{Acknowledgements}

Not applicable.

\section{Funding}

No funding was received.

\section{Availability of data and material}

The datasets generated and/or analyzed during the current study are available in the $\mathrm{dbGaP}$ (ncbi.nlm.nih.gov/gap) (NCBI dbGaP study accession: phs000699.v1.p1 and dbGaP analysis accession: pha003862).

\section{Authors' contributions}

YZ and FY made substantial contributions to the conception and design, and interpretation of the data. YZ and FY revised the manuscript critically for important intellectual content and gave final approval of the version to be published. YZ and FY agreed to be accountable for all aspects of the work in ensuring that questions related to the accuracy or integrity of any part of the study are appropriately investigated and resolved.

\section{Ethics approval and consent to participate}

Not applicable.

\section{Patient consent for publication}

Not applicable.

\section{Competing interests}

The authors declare that they have no competing interests.

\section{References}

1. Ottaviani $\mathrm{G}$ and Jaffe N: The epidemiology of osteosarcoma. Cancer Treat Res 152: 3-13, 2009.

2. Yang J, Cogdell D, Yang D, Hu L, Li H, Zheng H, Du X, Pang Y, Trent J, Chen K and Zhang W: Deletion of the WWOX gene and frequent loss of its protein expression in human osteosarcoma. Cancer Lett 291: 31-38, 2010.

3. Sparks AB, Peterson SN, Bell C, Loftus BJ, Hocking L, Cahill DP, Frassica FJ, Streeten EA, Levine MA, Fraser CM, et al: Mutation screening of the TNFRSF11A gene encoding receptor activator of NF kappa B (RANK) in familial and sporadic Paget's disease of bone and osteosarcoma. Calcif Tissue Int 68: 151-155, 2001.

4. Sadikovic B, Yoshimoto M, Chilton-MacNeill S, Thorner P, Squire JA and Zielenska M: Identification of interactive networks of gene expression associated with osteosarcoma oncogenesis by integrated molecular profiling. Hum Mol Genet 18: 1962-1975, 2009.

5. Ueda K, Cardarelli C, Gottesman MM and Pastan I: Expression of a full-length cDNA for the human 'MDR1' gene confers resistance to colchicine, doxorubicin, and vinblastine. Proc Natl Acad Sci USA 84: 3004-3008, 1987.

6. Jia M, Hu J, Li W, Su P, Zhang H, Zhang X and Zhou G: Trps1 is associated with the multidrug resistance of osteosarcoma by regulating MDR1 gene expression. FEBS Lett 588: 801-810, 2014.

7. Hu YS, Xin J, Hu Y, Zhang L and Wang J: Analyzing the genes related to Alzheimer's disease via a network and pathway-based approach. Alzheimers Res Ther 9: 29, 2017.

8. Cowley MJ, Pinese M, Kassahn KS, Waddell N, Pearson JV, Grimmond SM, Biankin AV, Hautaniemi S and Wu J: PINA v2.0: Mining interactome modules. Nucleic Acids Res 40 (Database Issue): D862-D865, 2012.

9. Menche J, Sharma A, Kitsak M, Ghiassian SD, Vidal M, Loscalzo J and Barabási AL: Disease networks. Uncovering disease-disease relationships through the incomplete interactome. Science 347: 1257601, 2015. 
10. Jia P, Zheng S, Long J, Zheng W and Zhao Z: dmGWAS: Dense module searching for genome-wide association studies in protein-protein interaction networks. Bioinformatics 27: 95-102, 2011.

11. Wang J, Duncan D, Shi Z and Zhang B: WEB-based GEne SeT AnaLysis Toolkit (WebGestalt): Update 2013. Nucleic Acids Res 41 (Web Server Issue): W77-W83, 2013.

12. Chen J, Bardes EE, Aronow BJ and Jegga AG: ToppGene Suite for gene list enrichment analysis and candidate gene prioritization. Nucleic Acids Res 37 (Web Server Issue): W305-W311, 2009.

13. Hu Y, Pan Z, Hu Y, Zhang L and Wang J: Network and pathway-based analyses of genes associated with Parkinson's disease. Mol Neurobiol 54: 4452-4465, 2017.

14. Shannon P, Markiel A, Ozier O, Baliga NS, Wang JT, Ramage D, Amin N, Schwikowski B and Ideker T: Cytoscape: A software environment for integrated models of biomolecular interaction networks. Genome Res 13: 2498-2504, 2003.

15. He $\mathrm{X}$ and Zhang J: Why do hubs tend to be essential in protein networks? PLoS Genet 2: e88, 2006.

16. Jeong H, Mason SP, Barabasi AL and Oltvai ZN: Lethality and centrality in protein networks. Nature 411: 41-42, 2001.

17. Yu H, Kim PM, Sprecher E, Trifonov V and Gerstein M: The importance of bottlenecks in protein networks: Correlation with gene essentiality and expression dynamics. PLoS Comput Biol 3: e59, 2007.

18. Wuchty S: Controllability in protein interaction networks. Proc Natl Acad Sci USA 111: 7156-7160, 2014.

19. Xing P, Liao Z, Ren Z, Zhao J, Song F, Wang G, Chen K and Yang J: Roles of low-density lipoprotein receptor-related protein 1 in tumors. Chin J Cancer 35: 6, 2016.

20. Yang J, Annala M, Ji P, Wang G, Zheng H, Codgell D, Du X, Fang Z, Sun B, Nykter M, et al: Recurrent LRP1-SNRNP25 and KCNMB4-CCND3 fusion genes promote tumor cell motility in human osteosarcoma. J Hematol Oncol 7: 76, 2014.

21. Shibayama T, Okamoto T, Nakashima Y, Kato T, Sakurai T, Minamiguchi S, Kataoka TR, Shibuya S, Yoshizawa A, Toguchida J and Haga H: Screening of BCOR-CCNB3 sarcoma using immunohistochemistry for CCNB3: A clinicopathological report of three pediatric cases. Pathol Int 65: 410-414, 2015.

22. Bousquet M, Noirot C, Accadbled F, Sales de Gauzy J, Castex MP, Brousset $\mathrm{P}$ and Gomez-Brouchet A: Whole-exome sequencing in osteosarcoma reveals important heterogeneity of genetic alterations. Ann Oncol 27: 738-744, 2016.

23. Saalfrank A, Janssen KP, Ravon M, Flisikowski K, Eser S, Steiger K, Flisikowska T, Müller-Fliedner P, Schulze É, Brönner C, et al: A porcine model of osteosarcoma Oncogenesis 5: e210, 2016.

24. Yotov WV, Hamel H, Rivard GE, Champagne MA, Russo PA, Leclerc JM, Bernstein ML and Levy E: Amplifications of DNA primase 1 (PRIM1) in human osteosarcoma. Genes Chromosomes Cancer 26: 62-69, 1999.

25. Xi Y and Chen Y: PTEN plays dual roles as a tumor suppressor in osteosarcoma cells. J Cell Biochem 118: 2684-2692, 2017.

26. Kersting C, Gebert C, Agelopoulos K, Schmidt H, van Diest PJ, Juergens H, Winkelmann W, Kevric M, Gosheger G, Brandt B, et al: Epidermal growth factor receptor expression in high-grade osteosarcomas is associated with a good clinical outcome. Clin Cancer Res 13: 2998-3005, 2007.

27. Mantovani FB, Morrison JA and Mutsaers AJ: Effects of epidermal growth factor receptor kinase inhibition on radiation response in canine osteosarcoma cells. BMC Vet Res 12: 82, 2016.

28. Deel MD, Li JJ, Crose LE and Linardic CM: A review: Molecular aberrations within hippo signaling in bone and soft-tissue sarcomas. Front Oncol 5: 190, 2015.
29. Niu NK, Wang ZL, Pan ST, Ding HQ, Au GH, He ZX, Zhou ZW, Xiao G, Yang YX, Zhang X, et al: Pro-apoptotic and pro-autophagic effects of the Aurora kinase A inhibitor alisertib (MLN8237) on human osteosarcoma U-2 OS and MG-63 cells through the activation of mitochondria-mediated pathway and inhibition of p38 MAPK/PI3K/Akt/mTOR signaling pathway. Drug Des Devel Ther 9: 1555-1584, 2015.

30. Li G, Cai M, Fu D, Chen K, Sun M, Cai Z and Cheng B: Heat shock protein $90 \mathrm{~B} 1$ plays an oncogenic role and is a target of microRNA-223 in human osteosarcoma. Cell Physiol Biochem 30: 1481-1490, 2012.

31. Ma J, Huang K, Ma Y, Zhou M and Fan S: The TAZ-miR-224-SMAD4 axis promotes tumorigenesis in osteosarcoma. Cell Death Dis 8: e2539, 2017.

32. Meng ZJ, Wu N, Liu Y, Shu KJ, Zou X, Zhang RX, Pi CJ, He BC, Ke ZY, Chen L, et al: Evodiamine inhibits the proliferation of human osteosarcoma cells by blocking PI3K/Akt signaling. Oncol Rep 34: 1388-1396, 2015.

33. Tingting R, Wei G, Changliang P, Xinchang L and Yi Y: Arsenic trioxide inhibits osteosarcoma cell invasiveness via MAPK signaling pathway. Cancer Biol Ther 10: 251-257, 2010.

34. Cai Y, Cai T and Chen Y: Wnt pathway in osteosarcoma, from oncogenic to therapeutic. J Cell Biochem 115: 625-631, 2014.

35. Du X, Yang J, Yang D, Tian W and Zhu Z: The genetic basis for inactivation of Wnt pathway in human osteosarcoma. BMC Cancer 14: 450, 2014.

36. Alkhalaf $M$ and Jaffal S: Potent antiproliferative effects of resveratrol on human osteosarcoma SJSA1 cells: Novel cellular mechanisms involving the ERKs/p53 cascade. Free Radic Biol Med 41: 318-325, 2006.

37. Deng Z, Liu X, Jin J, Xu H, Gao Q, Wang Y and Zhao J: Histone deacetylase inhibitor trichostatin a promotes the apoptosis of osteosarcoma cells through p53 signaling pathway activation. Int J Biol Sci 12: 1298-1308, 2016.

38. Sun Y, Xia P, Zhang H, Liu B and Shi Y: P53 is required for Doxorubicin-induced apoptosis via the TGF-beta signaling pathway in osteosarcoma-derived cells. Am J Cancer Res 6: 114-125, 2015.

39. Suzuki S and Kulkarni AB: Extracellular heat shock protein HSP90beta secreted by MG63 osteosarcoma cells inhibits activation of latent TGF-beta1. Biochem Biophys Res Commun 398: 525-531, 2010.

40. Zhou Y, Zhao RH, Tseng KF, Li KP, Lu ZG, Liu Y, Han K, Gan ZH, Lin SC, Hu HY and Min DL: Sirolimus induces apoptosis and reverses multidrug resistance in human osteosarcoma cells in vitro via increasing microRNA-34b expression. Acta Pharmacol Sin 37: 519-529, 2016.

41. Yu D, An F, He X and Cao X: Curcumin inhibits the proliferation and invasion of human osteosarcoma cell line MG-63 by regulating miR-138. Int J Clin Exp Pathol 8: 14946-14952, 2015.

42. Jiang R, Zhang C, Liu G, Gu R and Wu H: MicroRNA-126 inhibits proliferation, migration, invasion and EMT in osteosarcoma by targeting ZEB1. J Cell Biochem 118: 3765-3774, 2017.

43. Yun HM, Park KR, Quang TH, Oh H, Hong JT, Kim YC and Kim EC: 4-parvifuran inhibits metastatic and invasive actions through the JAK2/STAT3 pathway in osteosarcoma cells. Arch Pharm Res 40: 601-609, 2017.

This work is licensed under a Creative Commons Attribution-NonCommercial-NoDerivatives 4.0 International (CC BY-NC-ND 4.0) License. 